\title{
Relay Sensor Placement in Wireless Sensor Networks
}

\author{
Xiuzhen Cheng * Ding-Zhu Du ${ }^{\dagger}$ \\ Lusheng Wang $\ddagger$ Baogang $\mathrm{Xu}^{\S}$
}

\begin{abstract}
This paper addresses the following relay sensor placement problem: given the set of duty sensors in the plane and the upper bound of the transmission range, compute the minimum number of relay sensors such that the induced topology by all sensors is globally connected. This problem is motivated by practically considering the tradeoff among performance, lifetime, and cost when designing sensor networks. In our study, this problem is modelled by a NP-hard network optimization problem named Steiner Minimum Tree with Minimum number of Steiner Points and bounded edge length (SMT-MSP). We propose two approximate algorithms, together with their detailed performance analysis. The first one has performance ratio 3 and the second one has performance ratio 2.5 .
\end{abstract}

Keywords: Wireless sensor networks, relay sensor, Steiner point, SMT-MSP, topology control

*Department of Computer Science, the George Washington University, Washington, DC 20052. Email: cheng@gwu. edu.

${ }^{\dagger}$ Department of Computer Science and Engineering, University of Minnesota, Minneapolis, MN 55455, USA. Email: dzd@cs.umn.edu.

${ }_{\ddagger}^{\ddagger}$ Department of Computer Science, City University of Hong Kong, Kowloon, Hong Kong. E-mail: lwang@cs.cityu.edu.hk.

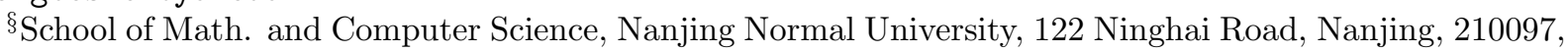
P.R. China. Email: baogxu@email.njnu.edu.cn. 


\section{Introduction}

Wireless Sensor Networks (WSNs) are ad hoc multihop systems containing sensors connected by wireless links. The flourishing research on WSNs is driven by the advances in MEMS technology, CMOS logic, and wireless networking $[5,10]$. WSNs have many possible applications, ranging from habitat monitoring to environment control [19]. WSN is used to produce macro-scale effects from micro-devices through coordinated activities of many sensors, thus connectivity is a very important issue in WSN architecture design.

Wireless links are mainly determined by transmit powers of sensors, and higher transmit power produces richer connectivity. However, "in the context of untethered nodes, the finite energy budget is a primary design constraint. Communications is a key energy consumer as the radio signal power in sensor networks drops off with $r^{4}$ [11] due to ground reflections from short antenna heights." (quoted from [4].) Here in this quote, $r$ is the distance from the transmitter. This means to reach a slightly longer distance, the sensor needs to dispatch much higher transmit power. The second reason for the prohibitiveness of higher transmit power is the higher interference to on-going traffic. The higher the power a sensor transmits, the more the number of direct neighbors the sensor has, and the higher the negative influence the sensor has on the network throughput. The third reason is the lifetime of the network [13], which is determined by the lifetime of sensors as a whole. Wireless sensors are battery powered [9]. Either battery renewal is prohibited by economic considerations or it is impossible to recharge or replace a battery in a WSN. The forth reason, but not the last, is the heat dissipated by higher-power transmission may meddle the sensing function (i.e. temperature sensors).

However, these observations do not mean that the lower the transmit power, the better. Very low transmit power may cause disconnected topology, and thus network malfunction. It may cause rocketing hop count for message dissemination, thus ascending error rate and falling throughput. Economically deploying a sensor network with very low transmit power may be prohibitive, since the number of sensors needed may be doubled or tripled. Therefore a sensor network designer has to seek a tradeoff among performance, lifetime, and cost. A number of problems have been formulated to study this tradeoff. Many of them focus on topology control by minimizing the maximum transmit power $[8,14]$ or minimizing total transmit power $[3,18]$ to maintain global topology. Our research focus is different. We consider the placement of relay sensors to connect all sensors on duty, called duty sensors, with fixed transmit powers.

Our study is motivated by an important class of wireless sensor networks, in which the locations (i.e. monitored sites) of the sensors are fixed and their placements are predetermined. Further, we assume all sensors are placed in a 2-dimensional plane. For example, in desertification monitoring, a set of sites in the desert are preselected and different kinds of environmental sensors are placed in each site. The sensor transmit power is pre-computed by comprehensively considering factors on network lifetime, performance, cost, etc. Since no sensor can reach the main office directly, individual observations at each site need to be directed through multihop relaying for further processing. Based on this consideration, we 
study the following problem: given a set of duty sensors (required sensors) in the plane, place minimum number of relay sensors to maintain global connectivity such that the transmission range of each sensor is at most $R$, where $R$ is a constant. This statement is modelled by the network optimization problem named Steiner Minimum Tree with Minimum number of Steiner Points and bounded edge length (SMT-MSP) [7]:

- Given a set of terminals (denoted by $V$ ) in the plane and a constant $R$, find a Steiner tree $\tau$ spanning $V$ with minimum number of Steiner points such that every edge in $\tau$ has length at most $R$.

In this description, "terminals" refer to "duty sensors" while "Steiner points" refer to "relay sensors". SMT-MSP is a generalized Steiner Minimum Tree (SMT) problem. A Steiner tree for terminal set $V$ is a spanning tree over $V \cup S$, where $S$ contains all points not in $V$, which are called Steiner points. A SMT is a Steiner tree with minimum total edge length.

The main reason for minimizing the number of Steiner points is to decrease system cost, as these relay sensors may have higher capability, thus higher cost. For example, in a desertification monitoring WSN, the relay sensors can be simple base stations with higher processing and relaying ability, compared with those environmental sensors. SMTMSP is NP-hard [7]. Lin and Xue [7] have proved that steinerized minimum spanning tree (adding minimum number of Steiner points on the edges of a minimum spanning tree to upper-bound the edge length to $R$ ) has performance ratio 5. The performance ratio of an approximate algorithm $\mathcal{A}$ to a minimization problem $\mathcal{P}$ is defined to be $\sup _{I} \frac{A_{I}}{O P T_{I}}$, where $I$ is an instance of problem $\mathcal{P}, A_{I}$ is the output from $\mathcal{A}$ for instance $I$, and $O P T_{I}$ is the optimal solution for instance $I$. In [2], Chen et al. show that steinerized Spanning tree actually has performance ratio exactly 4 . They also present a new $O\left(n^{4}\right)$-time approximate algorithm with performance ratio at most 3 , where $n$ is the number of given terminals. The time complexity of this algorithm, with a slight modification, is reduced to $O\left(n^{3}\right)$ in this paper. We also provide a randomized algorithm with performance ratio at most $\frac{5}{2}$.

In the following two sections we are going to propose two approximate algorithms ${ }^{1}$ for SMT-MSP, together with their theoretical performance analysis. We will briefly summarize the related work in Section 4 and conclude our paper in Section 5.

\section{A ratio 3 algorithm for STP-MSP}

Given a set $P$ of $n$ terminals in the Euclidean plane, and a positive constant $R$, we want to find a Steiner tree with minimum number of Steiner points such that each edge in the tree has length at most $R$. In [2], Chen et al. present an $O\left(n^{4}\right)$-time approximation with performance ratio at most 3 . With a slight modification, we may reduce the running time to $O\left(n^{3}\right)$.

Our algorithm is given in Figure 1. Since we construct 3-stars in Step 2, the algorithm runs in $O\left(n^{3}\right)$ time. Now we analyze this algorithm theoretically.

\footnotetext{
${ }^{1}$ We use terminals and Steiner points to refer to the duty sensors and relay sensors respectively.
} 


\section{Algorithm A: A 3-approximate Algorithm for STP-MSP}

Input: $\quad \mathrm{A}$ set $P$ of $n$ terminals, a positive constant $R$.

Output: A Steiner tree $T_{A}$ in which each edge has length at most $R$.

0 . Sort all $\frac{n(n-1)}{2}$ possible edges between the $n$ terminals of $P$ in length increasing order $e_{1}, e_{2}, \ldots, e_{\frac{n\left(n_{1}\right)}{2}}$, and set $T_{A}=(P, \emptyset)$;

1. for every $e_{i}$ such that $\left|e_{i}\right| \leq R$ do

if $e_{i}$ connects two different connected components of $T_{A}$

then put $e_{i}$ into $T_{A}$;

2. for each subset of three terminals $a, b, c$ respectively in three connected components of $T_{A}$ do

if there exists a point $s$ within distance $R$ from $a, b$ and $c$

then put the 3 -star, consisting of three edges $s a, s b, s c$, into $T_{A}$;

3. for every $e_{i}$ with $\left|e_{i}\right|>R$ in the increasing order do

if $e_{i}$ connects two different connected components of $T_{A}$

then put the steinerized $e_{i}$ into $T_{A}$.

Figure 1: The ratio-3 algorithm.

For a given set $P$ of terminals, a minimum spanning tree is a tree interconnecting the terminals in $P$ with edge between terminals. For a given constant $R$, a steinerized minimum spanning tree is a tree obtained from a minimum spanning tree by inserting $\left\lceil\frac{|a b|}{R}\right\rceil-1$ Steiner points to break each edge $a b$ into small pieces of length at most $R$. Edge $a b$ is a steinerized edge. A full component of a Steiner tree is a subtree in which each terminal is a leaf and each internal node is a Steiner point.

Let $T$ be a Steiner tree and $e$ be a line segment. $C(T)$ and $C(e)$ denote the numbers of Steiner points in $T$ and $e$, respectively. $|e|$ denotes the length of $e$.

Lemma 2.1 [2] Every steinerized minimum spanning tree has the minimum number of Steiner points among steinerized spanning trees.

Lemma 2.2 [7] There exists a shortest optimal Steiner tree $T^{*}$ for STP-MSP such that every vertex in $T^{*}$ has degree at most five.

Lemma 2.3 [2] Let $T^{*}$ be a shortest optimal tree for STP-MSP such that every Steiner point has degree at most five. Let $T_{j}$ be a full component of $T^{*}$. Then the following hold:

(1) The steinerized minimum spanning tree on terminals in $T_{j}$ has at most $3 \cdot C\left(T_{j}\right)+1$ Steiner points.

(2) If $T_{j}$ contains a Steiner point of degree at most four, then the steinerized minimum spanning tree on terminals in $T_{j}$ has at most $3 \cdot C\left(T_{j}\right)$ Steiner points. 
(3) If the steinerized minimum spanning tree on terminals in $T_{j}$ has an edge (of length at most $R$ ) between two terminals, then it contains at most $3 \cdot C\left(T_{j}\right)$ Steiner points.

From (3) of Lemma 2.3, we know that if the number of Steiner points contained in a steinerized spanning tree on terminals in a full component $T_{j}$ reaches the upper bound $3 \cdot C\left(T_{j}\right)+1$, then any two terminals are not connected directly by a single edge of length at most $R$, i.e., there must be a Steiner point between them.

Theorem 2.4 Let $T^{*}$ be an optimal tree for $S T P-M S P$ and $T_{A}$ an approximation produced by Algorithm A. Then $C\left(T_{A}\right) \leq 3 C\left(T^{*}\right)$.

Proof. Let $T^{S}$ be a steinerized minimum spanning tree on all terminals, and let $k$ be the number of 3-stars produced by Step 2 of Algorithm $A$. Then

$$
C\left(T_{A}\right) \leq C\left(T^{S}\right)-k
$$

By Lemma 2.2, we assume that each Steiner point of $T^{*}$ has degree at most five. Assume that $T^{*}$ has $h$ full components $T_{1}, T_{2}, \ldots, T_{h}$. For $i=1,2$, let $T^{(i)}$ be the components produced by Step $i$ of Algorithm $A$. We construct a steinerized spanning tree $T$ as follows: Initially, set $T:=T^{(1)}$, then for each full component $T_{j}(1 \leq j \leq h)$, add to $T$ the steinerized minimum spanning tree $H_{j}$ on terminals of $T_{j}$. If the resulted tree has a cycle, then destroy the cycle by deleting some edges of $H_{j}$. Without loss of generality, suppose that $T_{1}, T_{2}, \ldots, T_{g}$ $(g \leq h)$ are the full components in $T^{*}$ such that every Steiner point has degree five and $T^{(1)} \cup T_{j}$ has no cycle. Combining Lemma 2.1 and Lemma 2.3 with the fact that for destroying a cycle from $T \cup H_{j}$, a Steiner point must be removed unless $H_{j}$ contains an edge between two terminals, we have

$$
C\left(T^{S}\right) \leq C(T) \leq 3 C\left(T^{*}\right)+g,
$$

i.e.,

$$
C\left(T_{A}\right) \leq 3 C\left(T^{*}\right)+g-k .
$$

Suppose that $T^{(1)}$ has $p$ components. Then, $T^{(2)}$ has $p-2 k$ components $C_{1}, C_{2}, \ldots, C_{p-2 k}$. Now we construct another graph $H$ on all terminals as follows: Initially put all edges of $T^{(1)}$ into $H$, then consider every $T_{j}(1 \leq j \leq g)$. If $T_{j}$ has a unique Steiner point (this Steiner point connects five terminals which must lie in at most two $\left.C_{i}^{\prime} s\right)$, then among the five terminals there are three pairs (edges) of terminals, each pair (edge) lies in the same $C_{i}$. We add the three edges into $H$. If $T_{j}$ has at least two Steiner points, then there are two Steiner points each connecting four terminals, and we can also find three pairs (edges) of terminals such that each pair (edge) lies in the same $C_{i}$. Thus, we can add the three edges into $H$. It is clear that $H$ has at most $p-3 g$ components. Since each component of $H$ is contained by a $C_{i}$, we have $p-2 k \leq p-3 g$, then $g-k \leq \frac{3 g}{2}-k \leq 0$. This ends the proof. 


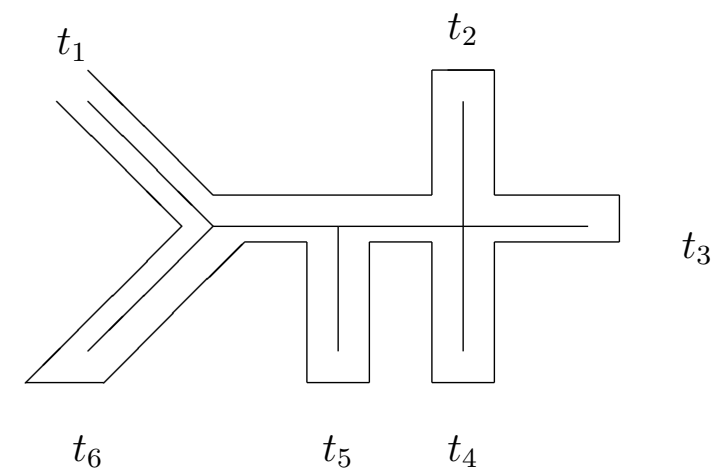

Figure 2: The tour $F$ that visits all the terminals

\section{A 2.5-approximate algorithm of STP-MSP}

In this section, we give a randomized algorithm of ratio- $\frac{5}{2}$ for the STP-MSP problem. The following are some useful terminologies and Lemmas.

A Steiner tree for $n$ terminals is a $k$-restricted Steiner tree if each full component spans at most $k$ terminals. A path $q_{1} q_{2} \ldots, q_{m}$ in a tree $T$ is called a convex path if for every $i=1,2, \ldots, m-3, q_{i} q_{i+2}$ intersects $q_{i+1} q_{i+3}$. An angle of degree more than $120^{\circ}$ is called a big angle. An angle of degree less than or equal to $120^{\circ}$ is called a small angle.

Lemma 3.1 [2] Let $q_{1} q_{2} \ldots, q_{m}$ be a convex path. Suppose there are $b$ big angles among $m-2$ angles $\angle q_{1} q_{2} q_{3}, \angle q_{2} q_{3} q_{4}, \ldots, \angle q_{m-2} q_{m-1} q_{m}$. Then, $\left|q_{1} q_{m}\right| \leq(b+2) R$.

Note that if there is no small angle, $\left|q_{1} q_{m}\right| \leq(b+1) R$. Thus this lemma is useful only when there are many small angles in the convex path.

Lemma 3.2 [2] In a shortest optimal tree $T$ for STP-MSP, there are at most two big angles at a point of degree three, there is at most one big angle at a point of degree four, and there is no big angle at a point of degree five.

Let $T^{*}$ be a full Steiner tree that is a shortest optimal tree for STP-MSP on $n$ terminals. Let $s_{i}$ denote the number of Steiner points of degree $i$ in $T^{*}$.

Lemma 3.3 [2] $3 s_{5}+2 s_{4}+s_{3}=n-2$.

Theorem 3.4 [12] There exists a randomized algorithm for the minimum spanning tree problem in 3-hypergraphs running in poly $\left(n, w_{\max }\right)$ time with probability at least 0.5 , where $n$ is the number of nodes in the hypergraph and $w_{\max }$ is the largest weight of edges in the hypergraph.

Lemma 3.5 Consider a clockwise tour $F$ of $T^{*}$ that visits the $n$ terminals in the order of $t_{1}, t_{2}, \ldots, t_{n}, t_{1}$ (see Figure 2). Then, 
(i) the tour $F$ has exactly $n$ convex paths $P_{1}, P_{2}, \ldots, P_{n}$ such that $P_{i}$ connects two terminals $t_{i}$ and $t_{i+1}\left(t_{n+1}=t_{1}\right)$;

(ii) each angle at a Steiner point appears in these $n$ convex paths exactly once.

(iii) connect the two ends of $P_{i}$ by an edge $e_{i}$ and then steinerize $e_{i}, i=1,2, \ldots, n$. The total number of Steiner points in any $n-1 e_{i}$ 's $\bar{C}=\sum_{i=1}^{n-1} C\left(e_{i}\right)$ is upper bounded as follows:

$$
\begin{aligned}
\bar{C} & \leq s_{4}+2 s_{3}+2 s_{2}+n-C\left(e_{n}\right) \\
& =3\left(s_{5}+s_{4}+s_{3}\right)+2 s_{2}+2-C\left(e_{n}\right)
\end{aligned}
$$

We denote by $T_{F}$ the tree consisting of $n$ terminals and $(n-1)$ edges $e_{1}, e_{2}, \ldots, e_{n-1}$.

Proof. (i) and (ii) are very easy to see from the structure of $T^{*}$. Now, we prove (iii). Consider the tour F. By Lemma 3.1, if there are $a_{i}$ big angles in $P_{i}$, then there are at most $a_{i}+1$ Steiner points on $e_{i}$, and so the total number of Steiner points in $F$ is at most $n$ plus the number of big angles in $T^{*}$. By Lemma 3.2, there are at most $2 s_{2}+2 s_{3}+s_{4}$ big angles in $T^{*}$. From Lemma 3.3, we know that (iii) is valid.

As defined previously, $T^{*}$ is a full Steiner tree that is a shortest optimal tree for STP-MSP on $n$ terminals. Without loss of generality, we assume that $T^{*}$ has Steiner points of degree at least three. Selecting an arbitrary Steiner point of degree at least three as the root of $T^{*}$, we get a rooted tree. A good point $t$ in $T^{*}$ is a Steiner point that is adjacent to some terminals and satisfies one of the following:

(i) (type (1)) $t$ has three or more terminals as children;

(ii) (type (2)) $t$ has two terminals as children and the degree of $t$ is 4 ;

(iii) (type (3)) $t$ is a point of degree 3 .

Note that a good point is of degree at least 3. A bad point is a Steiner point of degree at least 3 in $T^{*}$ that is not a good point.

Theorem 3.6 There is a 3-restricted Steiner tree such that each edge has length at most $R$ and the number of Steiner points is at most $\frac{5}{2}$ times the optimum.

Proof. Let $F$ and $T_{F}$ be defined as in Lemma 3.5. From Lemma 3.5, we know that in $T_{F}$, (1) each degree 2 Steiner point is used at most twice, and (2) each Steiner point of degree at least 3 is used at most three times.

We modify $T_{F}$ to get a 3 -restricted tree $T$ such that each of the good points of type (1) or type (3) in $T^{*}$ is used at most twice, at least half of the type (2) good points are used at most twice, and each of the rest of the Steiner points of degree at least 3 in $T^{*}$ is used at most three times.

Our modification is sketched below: 


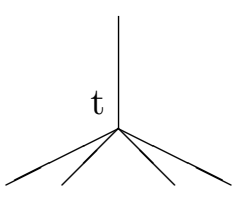

(a)

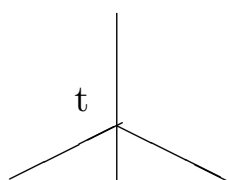

(b)

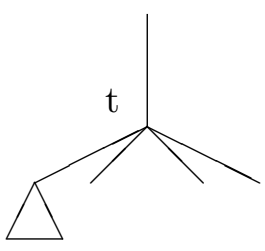

(c)

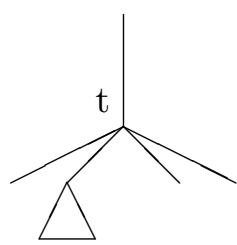

(d)

Figure 3: Four possible cases of type (1) good point

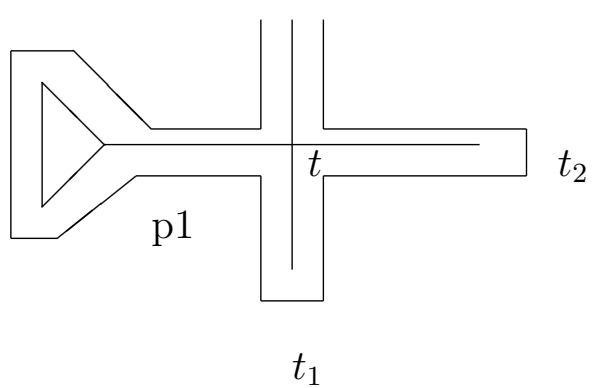

(a)

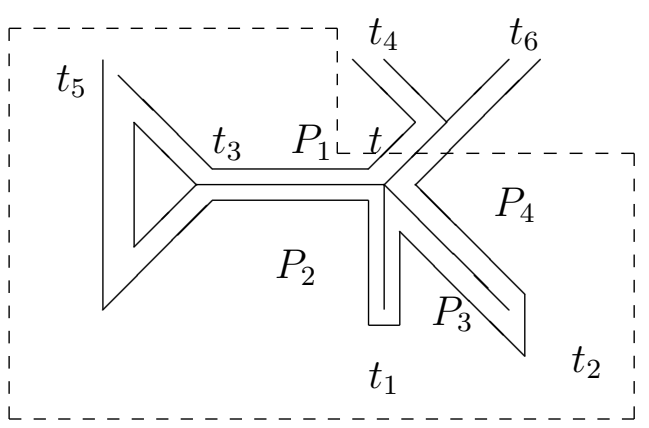

(b)

Figure 4: Two cases of type (2) good point. (a) Case 1. (b) Case 2.

(i) Let $t$ be a type (1) good point of degree $d$. We have to consider the four cases as shown in Figure 3.

There are $d$ convex paths in $F$ that go through $t$. We can use a 3 -star connecting the three terminals to replace two of the $d$ convex paths in the tour $F$. It is easy to verify that the number of times that $t$ is used in the new 3-restricted $T$ is reduced by 1 , i.e., $t$ is used at most twice (instead of three times).

(ii) Let $t$ be a type (2) good point. Thus, $t$ is of degree four. If there is no big angle at $t$, by Lemmas 3.1 and 3.2, the total number of big angles in $T^{*}$ is reduced by 1 , and $t$ is used at most twice. So, we can assume that there is a big angle at $t$.

Let $P_{1}$ be the convex path in the tour $F$ having the big angle at $t$. Let $t_{1}$ and $t_{2}$ be the two terminals adjacent to $t$. Two cases arise.

Case 1. $t_{1}$ or $t_{2}$, say, $t_{1}$, is in the convex path $P_{1}$ connecting $t_{1}$ and another terminal $t_{3}$ (see Figure $4(\mathrm{a})$ ). If $t_{3}=t_{2}$, then the convex path in $T_{F}$ connecting $t_{1}$ and $t_{2}$ has no small angle. Thus, the length $\left|t_{1} t_{2}\right| \leq(b+1) R$, i.e., the upper bound $(b+2) R$ in Lemma 3.1 is not tight. Therefore, the number of times that $t$ is used in $T_{F}$ is at most 2 (not 3 ). In this case, we do not have to modify the tree. If $t_{3} \neq t_{2}$, we connect $t_{2}$ to the convex path $P_{1}$ at point $t$. This forms a 3 -star with $t$ as the center connecting three terminals $t_{1}, t_{2}$ and $t_{3}$. We then use the three line segments $t t_{1}, t t_{2}$ and $t t_{3}$ (not the 3 convex paths) to form the three edges of the 3 -star. Let $t_{i}$ and $t_{j}$ be two points. We use $t_{i} t_{j}$ to represent the line segment 


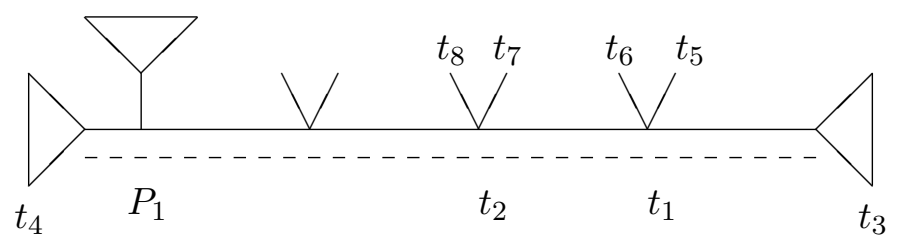

(a)

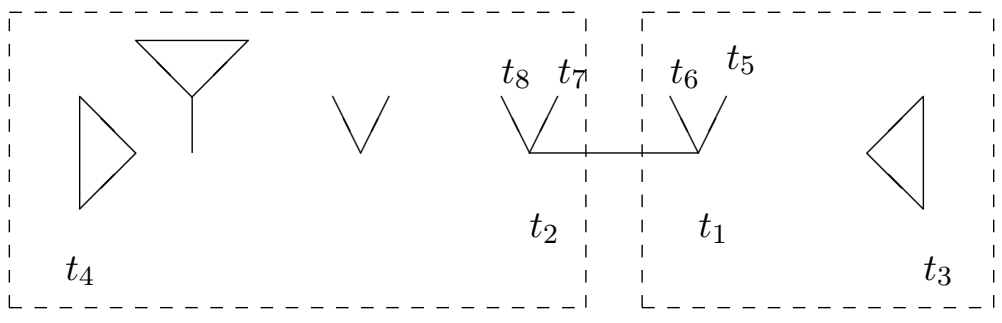

(b)

Figure 5: (a) The original $T^{*}$. The dashed line stands for $P_{1}$. (b) After deleting $P_{1}$, we have two components in the dashed boxes. We use an edge connecting $t_{1}$ and $t_{2}$ to form a tree again.

connecting $t_{i}$ and $t_{j}$. From Lemma 3.1, the big angle at $t$ ensures that

$$
C\left(t t_{1}\right)+C\left(t t_{3}\right)=C\left(t t_{3}\right) \text {. }
$$

Moreover, $C\left(t t_{2}\right)=b$ ( $t$ is not counted and there is no small angle in $\left.t t_{2}\right)$, where $b$ is the number of big angles in edge $t t_{2}$ in $T_{F}$. Note that $C\left(t_{1} t_{2}\right)$ is estimated as at least $b+1$ in Lemma 3.5. Thus, the number of times that $t$ is used in $T$ is reduced from at most 3 to at most 2 .

Case 2. Neither $t_{1}$ nor $t_{2}$ is in the convex path that has the big angle. Let $t_{3}$ be the child of $t$ other than $t_{1}$ and $t_{2}$. See Figure 4 (b). In this case, $t_{3}$ is either the leftmost child or the rightmost child of $t$. Without loss of generality, we assume that $t_{3}$ is the leftmost child, and let $P_{1}$ be the convex path in $F$ which connects two terminals $t_{4}$ and $t_{5}$ and contains the big angle at $t$ (see Figure $4(\mathrm{~b})$ ). Note that all the descendent terminals of $t$ (in the dashed circle in Figure 4 (b)) are connected with paths $P_{1}, P_{2}, P_{3}$ and $P_{4}$. Thus, we can shorten $P_{1}$ to obtain $P_{1}^{\prime}$ by cutting off the part from $t$ to $t_{5}$. By doing this, we get a 3 -star with $t$ as the center connecting $t_{1}, t_{2}$ and $t_{4}$. Since $P_{1}$ has a big angle at $t$, from Lemma 3.1, we know that the number of times that $t$ is used in $T$ is at most 2 (not 3 ).

In above discussion, we just consider the case where there is only one type (2) good point in the convex path $P_{1}$. Now, consider the case where there is more than one type (2) good point in $P_{1}$. See Figure 5 (a). Note that $T_{F}$ is a tree having $n-1$ edges (corresponding to convex paths) connecting the $n$ terminals. Let $e$ be the edge in $T_{F}$ which corresponds to $P_{1}$. Deleting the edge $e$ forms two components (inside a dashed box), with each containing an end of $e\left(t_{3}\right.$ or $\left.t_{4}\right)$. See Figure $5(\mathrm{~b})$. The terminals in each of the two components 


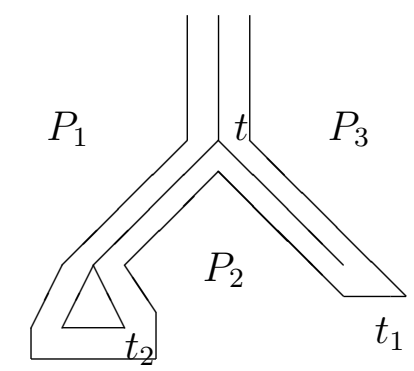

Figure 6: The type (3) Steiner point.

are connected by the rest of the $(n-2)$ edges (possibly replaced by some 3 -stars in the modification process).

Let $t_{1}$ and $t_{2}$ be the two type (2) good points in $P_{1}$ that are the leftmost and rightmost in the two components, respectively. See Figure 5 (b).

We replace $e$ by a segment (see the line in Figure 5 (b) connecting the two boxes) connecting $t_{1}$ and $t_{2}$ directly. Thus, we get a tree again. This makes all type (2) good points in $P_{1}$ other than $t_{1}$ and $t_{2}$ appear in $T$ at most twice (not three times). Moreover, we can form a 3 -star with $t_{1}$ as the center connecting $t_{5}, t_{6}$ and $t_{7}$, or with $t_{2}$ as the center connecting $t_{5}, t_{7}$ and $t_{8}$. By doing this, one of $t_{1}$ and $t_{2}$ appears in $T$ at most twice instead of three times.

Thus we can conclude that at least half of the type (2) good points are used in $T$ at most twice instead of three times.

(iii) $t$ has at least one child, say, $t_{1}$, and the degree of $t$ is 3 (see Figure 6). If there is at most one big angle at $t$, then in Lemma 3.5, $t$ is overestimated, i.e., $T$ is used in the tour $F$ at most twice (not three times). So, we assume that there are two big angles at $t$. Thus, at least one of the two convex paths $P_{2}$ and $P_{3}$, say, $P_{3}$, (See Figure 6,) has a big angle at $t$. We then can shorten the edge $e$ corresponding to $P_{3}$ in $T_{F}$ by cutting off the part from $t$ to $t_{1}$ and form a 3-star with $t$ as center connecting $t_{1}, t_{2}$ and $t_{3}$ (the other end of $P_{3}$ ). By doing this, we save at least one Steiner point, and thus the number of times that $t$ is used in $T$ is at most 2 (not 3$)$.

Note that in the above modification, we merge $P_{2}$ and $P_{3}$ into a 3 -star and save one Steiner point by taking the advantage of a big angle at $t$. Each convex path can only be used to form a 3 -star once. Otherwise, we get an $i$-star for $i>3$. Thus, we have to make sure that each type (3) good point $t$ can match a unique convex path that has a big angle at $t$. This can be done since each type (3) good point has degree 3 and there would be two big angles at $t$. (If there is only one or zero big angle at $t$, then $t$ is used only once or twice in $T_{F}$. Thus we do not have to do any modification.)

Consider the case that in the convex path $P_{1}$ there are many type $(2)$ good points and type (3) good points. Using the same argument as in (ii) demonstrated in Figure 5, we can replace the edge corresponding to $P_{1}$ in $T_{F}$ by the segment connecting $t_{1}$ and $t_{2}$ as in Figure 5. Thus, every type (2) and type (3) good point other than $t_{1}$ and $t_{2}$ is used at most twice 
(not three times). In this case, $t_{1}$ and $t_{2}$ compete the edge $t_{1} t_{2}$ to form a 3 -star. We assign $t_{1} t_{2}$ to either $t_{1}$ or $t_{2}$ using the following strategy: If a type (2) good point competes $t_{1} t_{2}$ with a type (3) good point, say, $t$, we always let the type (2) good point have $t_{1} t_{2}$ since the type (3) good point has another big angle at it, but the type (2) good point does not. If next time $t$ competes with another type (2) good point or type (3) good point, we let $t$ win. Thus, every type (3) good point appears at most twice in $T$ and at least half of the type (2) good points appear in $T$ at most twice.

Now, we can make sure that in $T$ each good point of type (1) and (3) appears at most twice, and at least half of the type (2) good points appear twice. From Lemma 3.5, we have

$$
C(T) \leq 2 g_{1}+2.5 g_{2}+3 b+2 s_{2}+2-C\left(e_{n}\right),
$$

where $g_{1}$ is the number of good points of type (1) and (3), $g_{2}$ is the number of type (2) good points, and $b$ is the number of bad points.

We can delete a convex path $P_{n}$ from tour $F$ to form $T_{F}$. We always delete the convex path such that the corresponding edge $e_{n}$ is the longest.

In the following, we show that $b<g_{1}$. Let $T^{\prime}$ be the tree obtained from $T^{*}$ by deleting all terminals. Obviously, each leave in $T^{\prime}$ is a good point. Therefore, the number of bad points is the number of points of degree at least 3 in $T^{\prime}$. Thus, $b<g_{1}$.

If $C\left(e_{n}\right) \geq 2$, we have

$$
C(T) \leq 2 g_{1}+2.5 g_{2}+3 b+2 s_{2}
$$

Therefore,

$$
C(T) \leq 2 g_{1}+2.5 g_{2}+2.5 b+0.5 b+2 s_{2} \leq 2.5 g_{1}+2.5 g_{2}+2.5 b+2 s_{2} \leq 2.5 C\left(T^{*}\right) .
$$

If $C\left(e_{n}\right)=1$, there is no big angle in $T^{*}$. Thus, there is no degree-3 point in $T^{*}$. Suppose that there are degree- 4 points in $T^{*}$. Since there is no big angle in $T^{*}$, the number of times of degree-4 Steiner points is overestimated. Thus (2) still holds.

Now, we only have to consider the case where each Steiner point in $T^{*}$ has degree 5 . In this case, each point in $T^{\prime}$ is either of degree 1 or degree at least 3 . Thus, the root of $T^{\prime}$ is either of degree 1 or degree at least 3 . In this case, it is easy to see that the number of leaves of $T^{\prime}$ is at least two more than the number of points of degree at least 3 , i.e., $g_{1} \geq b+2$. Therefore,

$$
C(T) \leq 2 g_{1}+2.5 g_{2}+2.5 b+0.5 b+2 s_{2}+1 \leq 2.5 g_{1}+2.5 g_{2}+2.5 b+2 s_{2} \leq 2.5 C\left(T^{*}\right) .
$$

Now, we focus on the computation of an optimal 3-restricted tree.

Let $H_{3}(V, F, W)$ be a weighted 3-hypergraph, where $V=P, F=\{(a, b) \mid a \in V$ and $b \in$ $V\} \cup\{(a, b, c) \mid a \in V, b \in V$ and $c \in V\}$, and for each edge $e \in F, w(e)$ is the smallest number of Steiner points to form an optimal solution of the STP-MSP problem on the terminals in $e$. 
Given three points $a, b$ and $c$ on the Euclidean plane, let $s$ be the Steiner point that minimizes $(|s a|+|s b|+|s c|)$, and let $k$ be the number of Steiner points in an optimum solution $T$ of STP-MSP on $\{a, b, c\}$ with constant $R$.

\section{Lemma 3.7}

$$
\left\lceil\frac{|s a|}{R}\right\rceil+\left\lceil\frac{|s b|}{R}\right\rceil+\left\lceil\frac{|s c|}{R}\right\rceil-2 \geq k \geq\left\lfloor\frac{|s a|}{R}\right\rfloor+\left\lfloor\frac{|s b|}{R}\right\rfloor+\left\lfloor\frac{|s c|}{R}\right\rfloor-2 .
$$

Proof. By Steinerizing the optimum Steiner tree, we get a solution of STP-MSP on $\{a, b, c\}$ with exactly $\left\lceil\frac{|s a|}{R}\right\rceil+\left\lceil\frac{|s b|}{R}\right\rceil+\left\lceil\frac{|s c|}{R}\right\rceil-2$ Steiner points.

Let $|T|$ be the total length of $T$, which is the sum of the length of edges of $T$. Then

$$
(k-1) \cdot R+3 R \geq|T| \geq|s a|+|s b|+|s c|,
$$

i.e.,

$$
k+2 \geq \frac{|s a|+|s b|+|s c|}{R} \geq\left\lfloor\frac{|s a|+|s b|+|s c|}{R}\right\rfloor \geq\left\lfloor\frac{|s a|}{R}\right\rfloor+\left\lfloor\frac{|s b|}{R}\right\rfloor+\left\lfloor\frac{|s c|}{R}\right\rfloor .
$$

Therefore, (3) holds.

Lemma 3.7 gives an upper bound on the cost of $(a, b, c)$.

Lemma 3.8 [17] Testing whether three circles has a point in common can be done in constant time.

For given points $a, b$ and $c$ on the Euclidean plane, one can find the minimum Steiner tree on $\{a, b, c\}$ in constant time. Let $q_{a, b, c}$ be the number of Steiner points used to steinerize the optimum Steiner tree on $\{a, b, c\}$, and $q_{P}=\max \left\{q_{a, b, c} \mid\{a, b, c\} \subset P\right\}$. Then, by Lemma 3.7 and Lemma 3.8, the weight $W$ of $H_{3}(V, F, W)$ can be calculated in $O\left(n^{3} q_{P}^{2}\right)$ time. By Theorem 3.4 and Theorem 3.6, we have

Theorem 3.9 Given a set $P$ of $n$ terminals and a positive constant $R$, there exists a randomized algorithm that computes a solution of STP-MSP on $P$ such that the number of Steiner points is at most $\frac{5}{2}$ times of the optimum running in poly $\left(n, q_{P}\right)$ time with probability at least 0.5 .

The complete algorithm is given in Figure 7.

\section{Related Work}

Note that our starting point on topology control is very different than those in the literature. We maintain global connectivity by introducing relay sensors to keep transmission range moderate while most related research works focus on algorithm design to control the 


\section{$\frac{5}{2}$-approximate Algorithm for STP-MSP}

Input: $\quad$ A set $P$ of $n$ terminals in the Euclidean plane, a positive constant $R$ Output: A 3-restricted Steiner tree $T$ in which each edge has length at most $R$.

1. Construct a weighted hypergraph $H_{3}(V, F, w)$.

2. Call the randomized algorithm in [12] to compute a minimum spanning tree $T$ for $H_{3}(V, F, w)$;

3. Replace every edge $f$ of the minimum spanning tree $T$ on $H_{3}(V, F, w)$ with a Steiner tree of $w(f)$ Steiner points such that the maximum length of each edge in the tree is at most $R$ and output the obtained tree.

Figure 7: The complete 2.5-approximate algorithm.

transmit power dissipated by each sensor [6][8][14][15][18]. For example, Ramanathan and Rosales-Hain [14] show that one can efficiently minimize the maximum per node transmit power and maintain global connectivity and biconnectivity. Rodoplu and Meng [15], and Wattenhofer et al. [18], propose heuristics independently with different assumption to minimize the total power from every sensor to a master site. Hu [6] first determines a topology according to Delaunay triangulation. Then the degree of each node is adjusted through neighbor negotiation such that each node has similar number of neighbors. Computing a minimum energy topology for a WSN is NP-hard [3]. Even though our starting point is different than works mentioned above, we claim that these techniques and our work can be combined coherently. For example, when designing a WSN, one can first apply any of the two algorithms proposed in this paper to compute all relay sensors based on restrictions on power dissipation or transmission range, then apply any of these techniques in literature based on given resources and optimization objectives to compute the global topology for further transmit power reduction.

An interesting work that also considers relay sensor placement is reported in [16]. This work computes the minimum set of relay nodes such that each sensor can connect to 1 or 2 relay nodes and all relay nodes are either connected or 2-connected. In other words, the algorithms in [16] consider a hierarchical topology while our work sticks to the flat topology. Sensor placement that induces regular topologies such as circular, star, and hexagonal are presented and analyzed in [1].

\section{Conclusion and Future Work}

This paper tackles the problem of computing relay sensors to maintain global connectivity in WSNs when transmission range of all sensors are restricted. Our objective is to minimize the 
number of relay sensors needed, as they contribute to the overall cost of a WSN. We model this problem by a network optimization problem named SMT-MSP - Steiner Minimum Tree with Minimum number of Steiner Points and bounded edge length. SMT-MSP is NP-Complete. We propose two approximate algorithms for SMT-MSP and give detailed theoretical performance analysis.

Note that our algorithms in this paper compute relay sensors to globally network all duty sensors. As a future work we will consider the optimal relay sensor placement for $k$-connectivity, where $k>1$, to improve fault tolerance in sensor networks. We also will consider the design tradeoff between transmit power per sensor and the number of sensors in the network for topology control.

\section{References}

[1] E.S. Biagioni and G. Sasaki, Wireless sensor placement for reliable and efficient data collection, IEEE HICSS'03, Big Island, Hawaii, 2003.

[2] D. Chen, D.-Z. Du, X.. Hu, G. Lin, L. Wang and G. Xue, "Approximations for Steiner trees with minimum number of Steiner points", Journal of Global Optimization, vol. 18, pp. 17-33, 2000.

[3] X. Cheng, B. Narahari, and R. Simha, M. X. Cheng, and D. Liu, Strong minimum energy topology in wireless sensor networks: NP-completeness and heuristics, IEEE Transactions on Mobile Computing, vol. 2, no. 3, pp. 248-256, July-September 2003.

[4] D. Estrin, L. Girod, G. Pottie, and M. Srivastava, Instrumenting the world with wireless sensor networks, Proceedings of International Conference on Acoustics, Speech, and Signal Processing, vol. 4, pp. 2033-2036, 2001.

[5] J. Hill, R. Szewczyk, A. Woo, S. Hollar, D. Culler, and K. Pister, System architecture directions for networked sensors, ACM ASPLOS-IX, 2000, Cambridge, MA.

[6] L. Hu, Topology control for multihop packet radio networks, IEEE Transactions on Communications, vol. 41(10), pp. 1474-1481, 1993

[7] G. Lin and G. Xue, "Steiner tree problem with minimum number of Steiner points and bounded edge-length", Information Processing Letters, 69, pp. 53-57, 1999.

[8] E.L. Lloyd, R. Liu, M.V. Marathe, R. Ramanathan and S.S. Ravi, Algorithmic Aspects of Topology Control Problems for ad hoc networks, MobiCom 2002, pp. 123-134, 2002.

[9] R. Min, M. Bhardwaj, S.-H. Choi, N. Ickes, E. Shih, A. Sinha, A. Wang and A. Chandrakasan, Energy-centric enabling technologies for wireless sensor networks, IEEE Wireless Communications, pp. 28-39, August 2002.

[10] G.J. Pottie, Wireless sensor networks, Information Theory Workshop, pp. 139-140, 1998 
[11] G. Pottie and W. Kaiser, Wireless sensor networks, Communications of the ACM, vol. 43 (5), pp. 51-58, May 2000

[12] H.J. Prömel and A. Steger, "A New Approximation Algorithm for the Steiner Tree Problem with Performance Ratio 5/3", Journal of Algorithms, 36, pp. 89-101, 2000.

[13] V. Raghunathan, C. Schurgers, S. Park, and M. Srivastava, Energy-aware wireless sensor networks, IEEE Signal Processing, Vol. 19 (2), pp. 40-50, 2002.

[14] R. Ramanathan and R. Rosales-Hain, Topology control of multihop wireless networks using transmit power adjustment, INFOCOM 2000, vol. 2, pp. 404-413, 2000

[15] V. Rodoplu and T.H. Meng, Minimum energy mobile wireless networks, IEEE J. Selected Areas in Communications, vol. 17(8), pp. 1333-1344, August, 1999

[16] J. Tang, B. Hao, and A. Sen, Relay node placement in large scale wireless sensor networks, manuscript, 2004.

[17] L. wang and Z. Li, An Approximation Algorithm for a Bottleneck Steiner Tree Problem in the Euclidean Plane, submitted.

[18] R. Wattenhofer, L. Li, P. Bahl, and Y.-M. Wang, Distributed topology control for power efficient operation in multihop wireless ad hoc networks, INFOCOM 2001, vol 3, pp. 1388-1397, 2001

[19] N. $\mathrm{Xu}, \quad \mathrm{A}$ Survey of Sensor Network Applications, http://enl.usc.edu/ ningxu/papers/survey.pdf 